\title{
Internet das Coisas aplicada à Educação: Um Mapeamento Sistemático
}

\author{
Raul Donaire Gonçalves Oliveira ${ }^{1}$, Venilton FalvoJr ${ }^{1}$, Ellen Francine Barbosa ${ }^{1}$ \\ ${ }^{1}$ Instituto de Ciências Matemáticas e de Computação (ICMC-USP) \\ Caixa Postal 668 - CEP 13560-970 - São Carlos (SP), Brasil \\ raul.oliveira@usp.br, falvojr@usp.br, francinedicmc.usp.br
}

\begin{abstract}
Resumo. A Internet das Coisas (IoT) tem proporcionado soluções para problemas em diversas áreas, dentre as quais a educação destaca-se como uma vertente atual e relevante. Neste artigo são sintetizados os resultados de um Mapeamento Sistemático (MS) conduzido com o objetivo de avaliar o estado da arte na área de IoT aplicada à educação. Entre os resultados obtidos, identificou-se uma relação recorrente da adoção de IoT com foco interdisciplinar e, em especial, no ensino de disciplinas nas áreas de exatas e línguas. No que se refere às tecnologias adotadas, foi possivel observar a predominância do uso de dispositivos móveis e RFID tags no desenvolvimento de aplicações IoT para educação. Por outro lado, também foram identificadas lacunas referentes à avaliação das aplicações desenvolvidas. A principal contribuição deste estudo consiste em apresentar uma visão geral da adoção de IoT na educação, proporcionando um direcionamento em relação às principais tendências e lacunas de pesquisa que requerem maior atenção por parte dos pesquisadores, em curto e médio prazo.
\end{abstract}

\section{Introdução}

Internet das Coisas (Internet of Things, ou IoT) é uma das áreas emergentes da computação moderna. Considerada como uma revolução tecnológica, sua premissa está baseada em que objetos do nosso dia-a-dia (isto é, as "coisas") podem conectar-se e comunicar-se por meio de uma rede, possuindo endereço próprio e, assim, sendo identificados individualmente [Atzori et al. 2010].

Esta tecnologia possui aplicações em diversas áreas, saúde [Chandel et al. 2016], indústria [Correndo et al. 2016] e segurança [Aydin and Othman 2017]. Outra área de destaque na qual IoT pode ser aplicada é a educação. Segundo Tripathi [Tripathi 2016], IoT já está revolucionando a educação de diversas maneiras: (i) conectando universidades pelo mundo todo, (ii) criando um ambiente mais seguro para o ensino, (iii) aumentando o alcance do conhecimento, e (iv) despertando nos estudantes o desejo da pesquisa e desenvolvimento.

Vários educadores, apoiados pelas pesquisas conduzidas na área educacional, estão encontrando em IoT uma maneira de expandir seus horizontes e criar novas abordagens experimentais de ensino e aprendizagem. Nesse sentido, uma visão geral sobre a área é fundamental para aqueles que desejam conhecer melhor quais são os avanços e os desafios relacionados à adoção de IoT no cenário educacional.

Este trabalho visa identificar e discutir estudos que caracterizem o estado da arte de aplicações de IoT na educação. É nessa perspectiva que a condução de um Mapeamento Sistemático (MS) sobre o assunto representa uma atividade de fundamental re- 
levância. Em linhas gerais, mapeamentos sistemáticos são estudos detalhados, cujo principal objetivo é a busca por respostas em uma determinada área, procurando diminuir ao máximo o viés do pesquisador de modo a garantir uma resposta fiel e condizente com a realidade [Kitchenham and Charters 2007, Petersen et al. 2015].

Dentro desse contexto, almeja-se responder às seguintes questões: (i) quais são as principais áreas de conhecimento onde IoT vem sendo aplicada como apoio ao ensino e aprendizagem; (ii) quais são as principais tecnologias utilizadas no desenvolvimento de aplicações de IoT na educação; e (iii) como tais aplicações vêm sendo desenvolvidas.

Este trabalho está organizado como se segue. Na Seção 2 é apresentado como o MS foi conduzido e quais decisões foram tomadas durante sua execução. Na Seção 3 é apresentada uma análise dos resultados obtidos, bem como uma síntese de alguns dos estudos considerados mais interessantes sobre o tema. Por fim, na Seção 4 são apresentadas as principais conclusões e perspectivas para a continuidade do trabalho.

\section{Método}

Neste MS, o principal objetivo de pesquisa é identificar quais são os estudos primários mais relevantes que discutem o uso de IoT na educação. Especificamente, pretende-se identificar quais são as principais áreas de conhecimento em que IoT vem sendo utilizado como apoio ao ensino e aprendizagem e quais tecnologias estão sendo utilizadas no desenvolvimento de aplicações IoT no ambiente educacional. Além disso, também é importante discutir como essas aplicações estão sendo avaliadas, ou seja, identificar se existem métodos de avaliação que ajudem a checar a qualidade das aplicações IoT desenvolvidas. Para alcançar o objetivo de pesquisa definido, três questões de pesquisa (QP) foram estabelecidas:

- QP1: Quais são as principais áreas de conhecimento em que IoT vem sendo utilizado como apoio a atividades de ensino e aprendizagem?

- QP2: Quais são as principais tecnologias utilizadas no desenvolvimento de aplicações de IoT na educação?

- QP3: Quais são os principais métodos utilizados na avaliação de aplicações de IoT na educação?

A fim de responder a essas questões de pesquisa, foi definida como string de busca: "IoT OR Internet of Things AND learning $O R$ teaching $O R$ educational $O R$ education". Os mecanismos de busca utilizados (ACM Digital Library, El Compendex, IEEE Digital Library, ISI Web of Science, Science@Direct, Scopus) foram escolhidos considerando sua relevância.

Para os estudos selecionados, foi utilizado como critério de inclusão:

- CI1: O estudo primário aborda aplicações práticas de IoT na educação.

Em contrapartida, foram estabelecidos como critérios de exclusão:

- CE1: O estudo primário não aborda aplicações práticas de IoT na educação.

- CE2: O estudo primário apresenta uma visão superficial e/ou pouca contribuição significativa relacionando IoT e educação.

- CE3: O texto completo do estudo primário está inacessível.

- CE4: O estudo primário faz parte da literatura cinza (relatórios, avaliações ou quais outros tipos de documentos não publicados). 
Aplicando a string de busca nas bases de dados, 3299 estudos primários foram retornados, dos quais 1638 estudos eram duplicados. Assim, 1661 estudos foram considerados para análise. Dos 1661 estudos restantes foram analisados: título, resumo e palavras-chave. Após a leitura de cada item, foram aplicados os critérios de inclusão e exclusão. Como resultado, foram rejeitados 1475 estudos e 186 foram selecionados. A maioria dos estudos primários foram excluídos pelo critério de exclusão CE1.

A partir dos 186 estudos selecionados, foi realizado um segundo refinamento, desta vez incluindo na análise a leitura da seção introdutória do estudo. Após este processo, outros 137 estudos foram excluídos, principalmente pelo critério de exclusão CE2. Restaram, assim, 49 estudos potencialmente relevantes.

Em uma última seleção, todos os estudos primários restantes foram submetidos à leitura completa. Cada artigo foi analisado novamente, considerando os critérios de inclusão e exclusão. Potenciais dúvidas foram discutidas com especialista da área. Como resultado, 33 estudos foram aprovados e 16 estudos foram rejeitados. A lista completa de estudos selecionados pode ser encontrada em https: / / imgur. com/a/4X71B2x.

\section{Análise de Dados e Resultados}

Nesta seção é apresentada uma análise dos dados extraídos a partir da condução do MS, a fim de responder às QPs estabelecidas anteriormente.

A Figura 1 ilustra a distribuição temporal das publicações dos estudos. Foram considerados trabalhos publicados até meados de 2017. Como é possível observar, houve um crescimento na popularidade do tema no decorrer dos últimos anos, sendo que o ano com a maior quantidade de trabalhos associados foi 2016. No entanto, apesar do crescimento observado, é importante ressaltar que ainda são poucos os trabalhos realizados envolvendo IoT na educação.

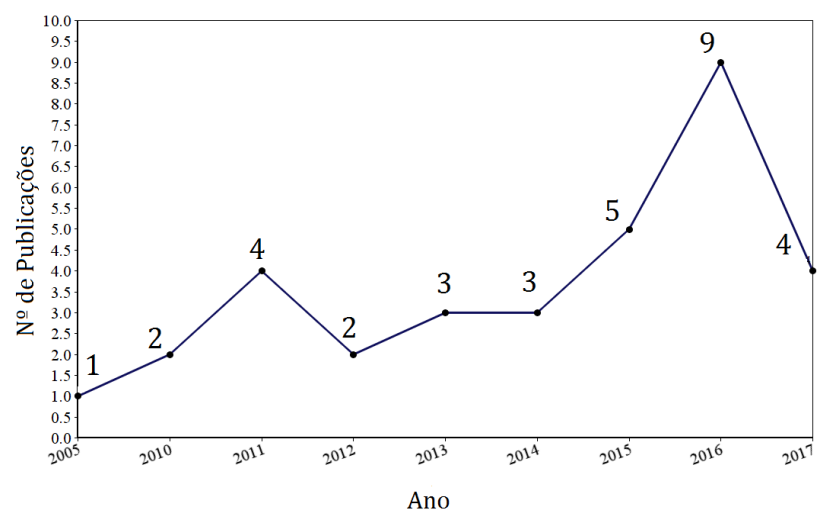

Figura 1. Número de Estudos por Ano

Durante a leitura dos estudos selecionados, também foi possível identificar uma clara divisão no foco dos estudos. A maioria dos estudos podem ser divididos em dois grupos: (i) estudos com foco educacional; e (ii) estudos com foco técnico. Nos estudos com foco educacional, os autores assumiram predominantemente o papel de educadores, enfatizando no estudo os aspectos educacionais do seu trabalho (abordagens e práticas de ensino, áreas de conhecimento, feedback dos alunos e professores). De modo geral, tais estudos possibilitaram melhores respostas à QP1. Já nos estudos com foco técnico, os autores assumiram o papel de desenvolvedores, focando em aspectos tecnológicos de 
VIII Congresso Brasileiro de Informática na Educação (CBIE 2019)

Anais do XXX Simpósio Brasileiro de Informática na Educação (SBIE 2019)

suas aplicações (detalhando algoritmos, benchmarks, dispositivos utilizados), facilitando na identificação das respostas à QP2. Os trabalhos mais siginficativos, representantes de cada um destes grupos, são apresentados nas respostas às questões de pesquisa, discutidas a seguir.

\subsection{Quais são as principais áreas de conhecimento em que IoT vem sendo utilizada como apoio a atividades de ensino e aprendizagem?}

A principal motivação associada à esta questão de pesquisa é identificar quais áreas de conhecimento têm recebido maior atenção da comunidade com respeito à utilização de IoT na educação e, em contrapartida, identificar também os domínios que estão sendo pouco investigados.

A Figura 2 sumariza os resultados obtidos com respeito à QP1. Pode-se observar que as contribuições da maioria dos trabalhos são interdisciplinares $(37,5 \%)$, ou seja, sua aplicabilidade estende-se a diferentes domínios de conhecimento (Ciências Exatas, Biológicas ou Humanas).

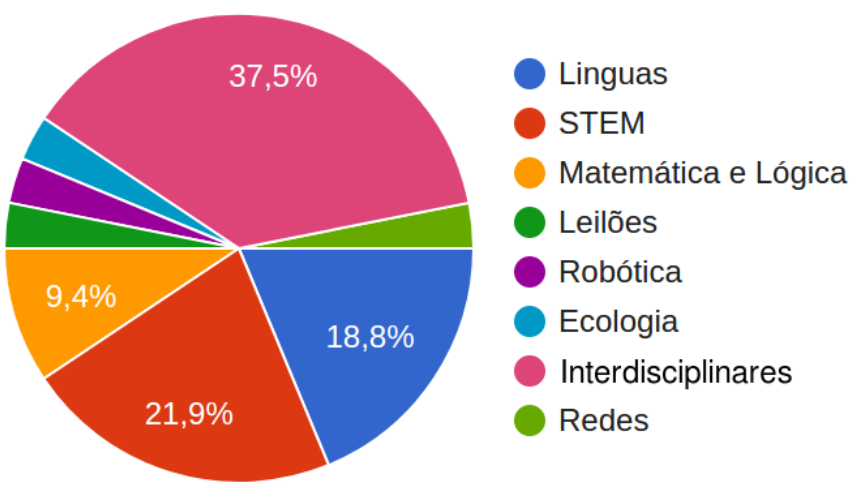

Figura 2. Figura 4. QP1: Áreas de Conhecimento

Um exemplo de trabalho aplicável a qualquer domínio é a lousa interativa, sugerida por Feng-Cheng Chang [Chang et al. 2015]. Em seu trabalho, o autor descreve todas as tecnologias e implementações necessárias para a construção de uma lousa que possa ser controlada remotamente por meio de um tablet. A ideia é que tal tecnologia possa ser utilizada em praticamente qualquer disciplina, de qualquer curso, visando a proporcionar uma aula mais dinâmica e interessante.

As disciplinas STEM (Science, Technology, Engineering and Mathematics) também apresentaram uma alta ocorrência de trabalhos abordando o uso de IoT na educação, totalizando $21,9 \%$ do número total de estudos retornados.

No trabalho de Charlton e Avramides [Charlton and Avramides 2016], por exemplo, os autores conduziram um experimento para avaliar uma abordagem de Aprendizado Baseada em Problemas para o ensino de disciplinas STEM, sugerindo IoT como principal tecnologia associada.

No experimento, os autores dividiram uma turma de alunos (entre 14 e 15 anos de idade) em pequenos grupos. Em um primeiro momento, foi ministrada uma aula em que os conceitos principais de IoT foram ensinados, exemplificando os benefícios que esta 
VIII Congresso Brasileiro de Informática na Educação (CBIE 2019)

Anais do XXX Simpósio Brasileiro de Informática na Educação (SBIE 2019)

tecnologia pode proporcionar à sociedade. Em seguida, iniciou-se uma discussão sobre como IoT poderia melhorar variados aspectos do cotidiano dos alunos, incentivando-os a apresentar soluções que utilizassem os conceitos recém-vistos. Cada grupo escolheu uma solução com o intuito de desenvolver, em conjunto, um protótipo (uma aplicação IoT que simulasse especificações reais). Ao final, todos os grupos compartilharam suas ideias e progressos em um workshop.

Entre os trabalhos desenvolvidos pelos alunos destacam-se uma luva inteligente smart glove, capaz de controlar aparelhos domésticos, e um robô capaz de auxiliar pessoas cegas a se locomoverem. Baseado nos resultados obtidos e no feedback dos alunos, os autores também observaram que IoT atuou como um importante impulsionador para a Aprendizagem Colaborativa.

Outra área bastante investigada a partir dos estudos retornados pelo MS referese ao ensino de línguas $(18,8 \%)$. Foram incluídas nesta categoria diversas respostas que visavam, de alguma forma, auxiliar o aprendizado de qualquer tipo de idioma. Nesse sentido, foram considerados desde tradutores, câmeras para verificar se a pronúncia das palavras estavam corretas [Wang 2010] e até mesmo cartões com microchips contendo o nome de figuras em diversas línguas [de la Guía et al. 2016].

Um exemplo de trabalho na área foi o realizado por Meda et al. [Meda et al. 2014]. Nele, os autores utilizaram as premissas da Aprendizagem Ubíqua (U-learning), desenvolvendo um aplicativo para dispositivos móveis capaz de traduzir em tempo real qualquer texto em telugo (idioma nativo indiano) para o inglês. Para utilizar o aplicativo o usuário precisava tirar uma foto do escrito; o arquivo era então enviado através da internet e processado em um servidor utilizando um algoritmo Google de tradução. A tradução retornava diretamente para a tela do usuário, juntamente com termo original para serem comparados.

\subsection{Quais são as principais tecnologias utilizadas no desenvolvimento de aplicações de IoT na educação?}

Esta questão de pesquisa visa identificar, dentre as aplicações educacionais encontradas, quais são as principais tecnologias utilizadas no desenvolvimento de aplicações relacionadas à IoT na educação. A motivação é identificar os arcabouços tecnológicos mais utilizados considerando o estado da arte.

Como pode ser observado na Figura 3, o uso de dispositivos móveis e RFID foram os resultados mais presentes nos estudos retornados. A principal razão para a significativa presença destas tecnologias refere-se ao custo e à facilidade de acesso, o que muitas vezes indica um baixo investimento relativo para a implementação de aplicações educacionais [dos Santos et al. 2016]. Na categoria "Outros" foram incluídas todas as tecnologias que apareceram em apenas um estudo.

No trabalho de M. Muñoz-Organero et al. [Muñoz-Organero et al. 2011] tem-se um exemplo de aplicação utilizando dispositivos móveis em conjunto com tags RFID. O objetivo era ministrar uma aula sobre Redes de Computadores que proporcionasse maior engajamento e interação dos alunos com o conteúdo. Para isso, os autores utilizaram as tags RFID como rótulos para diversas peças de hardware relacionadas ao tema. Para descobrirem o nome e o propósito de cada peça, os alunos precisavam fazer uso de seus celulares, os quais captavam as tags por meio de bluetooth, exibindo em seu display todas as informações disponíveis. A partir do experimento realizado, os autores concluíram que a aula experimental foi mais eficiente que o modelo tradicional, sendo IoT o grande 
VIII Congresso Brasileiro de Informática na Educação (CBIE 2019)

Anais do XXX Simpósio Brasileiro de Informática na Educação (SBIE 2019)

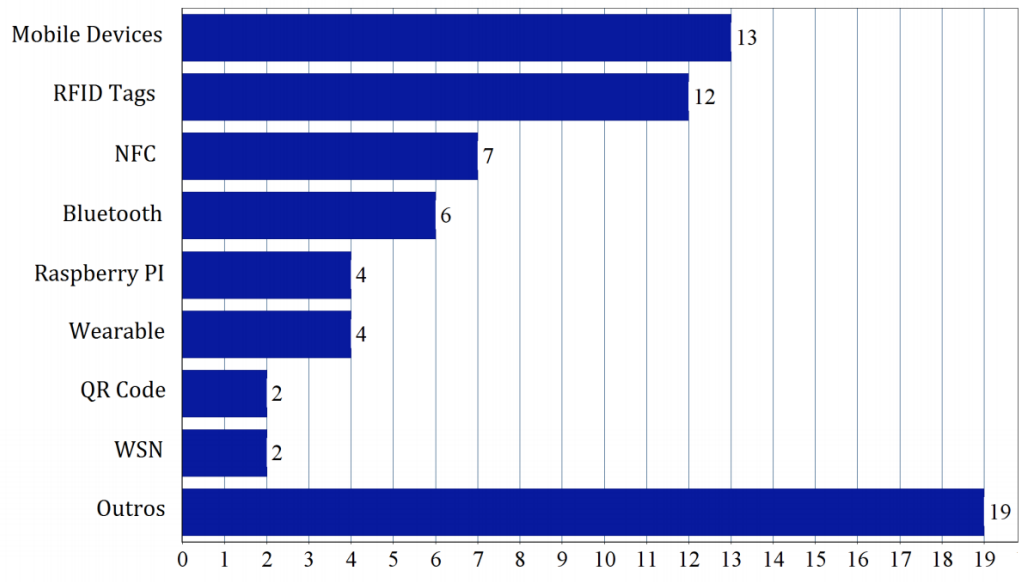

Figura 3. Tecnologias loT Encontradas

diferencial para a melhoria do aprendizado.

Outro exemplo de tecnologia RFID foi reportado no trabalho de Miglino et al. [Miglino et al. 2015]. Os autores descreveram a ferramenta Blockmagic, que permite que crianças interajam com o brinquedo Logic Blocks, conectados a um computador, com a intenção de estimular diversas atividades de aprendizado em habilidades específicas envolvendo matemática, lógica, línguas, resolução de problemas e criatividade. Todos os blocos foram rotulados com tags RFID, possuindo características distintas (cores, formatos, espessuras). Quando em contato com a ferramenta, as tags eram reconhecidas por sensores e esta interação era exibida na tela do computador, sendo possível designar tarefas e reconhecer o progresso da criança. O estudo foi aplicado em países da Europa e os autores chegaram à conclusão de que Blockmagic é uma ferramenta que poderia ser fácilmente introduzida à rotinas diárias de uma sala de aula. As crianças com necessidades especiais demonstraram grande aceitação pelo método, inclusive reduzindo o feedback negativo, quando comparado aos métodos tradicionais.

IoT também esteve presente como um elemento impulsionador para tecnologia assistiva, como pode ser observado no trabalho de Sula et al. [Sula et al. 2013]. Em sua pesquisa, os autores desenvolveram uma aplicação voltada ao ensino básico de lógica e linguagem, visando melhorar de qualidade de vida de crianças com Transtorno do Espectro Autista. A aplicação, chamada de Smartbox, faz uso de comunicação peer to peer (P2P) entre as crianças e seus cuidadores, proporcionando experiências interessantes de aprendizado. Em linhas gerais, a Smartbox permite que a criança interaja com objetos, sons, desenhos, fotografias e textos e, com o auxílio do sistema computacional, desenvolva habilidades de lógica, fala e demais conhecimentos necessários para sua melhor convivência cotidiana.

A tecnologia WSN (Wireless Sensor Network) foi identificada em dois estudos selecionados, sendo um deles a plataforma desenvolvida por Wang et al. [Wang et al. 2016]. Com o intuito de fomentar a conscientização ecológica, os autores desenvolveram uma ferramenta capaz de monitorar o nível de toxicidade de um lago local. A ferramenta faz uso dessa rede de sensores para captar tais informações e divulgar em tempo real a situação em um website. As informações coletadas são utilizadas em aulas de biologia 
VIII Congresso Brasileiro de Informática na Educação (CBIE 2019)

Anais do XXX Simpósio Brasileiro de Informática na Educação (SBIE 2019)

nas escolas da região.

No trabalho feito por Santos et al. [dos Santos et al. 2016], os autores descrevem como foi a experiência de utilizar o kit LEGO Mindstorms e placas Arduino para implementação de um laboratório de robótica destinado a alunos do ensino básico. A proposta foi utilizar estes mecanismos, juntamente com uma metodologia baseada em projetos, para aprendizagem de conceitos de robótica e automação. Os pesquisadores observaram um aumento significativo no interesse dos alunos em disciplinas relacionadas à matemática, física e programação, graças ao seu constante envolvimento prático no processo de desenvolvimento dos robôs.

Por fim, Bluetooth, dispositivos móveis e diversos sensores (temperatura, umidade, CO2) foram utilizados por Uzelac et al. [Uzelac et al. 2015] para a implementação de uma Smart Classroom. A proposta foi fazer uso desses dispositivos para descobrir o quanto parâmetros externos podem prejudicar a atenção dos alunos. Os autores confirmaram que, a partir dos dados coletados, é possível a criação de uma Smart Classroom capaz de perceber, em tempo real, se determinada turma está ou não focada na aula sendo ministrada.

\subsection{Quais são os principais métodos utilizados na avaliação de aplicações de IoT na educação?}

o objetivo desta questão de pesquisa foi identificar quais são os principais métodos de avaliação utilizados na verificação da eficácia e eficiência das aplicações que unem IoT e educação.

A Figura 4 sumariza os resultados encontrados. De modo geral, pode-se observar a predominância de avaliações quantitativas, presente em 13 dos estudos selecionados. Por outro lado, também foi possível constatar que parte dos trabalhos identificados não conduziram nenhum tipo de avaliação das aplicações desenvolvidas. De um total de 33 estudos, 12 não conseguiram responder a esta questão de pesquisa por não possuírem nenhum método de avaliação associado.

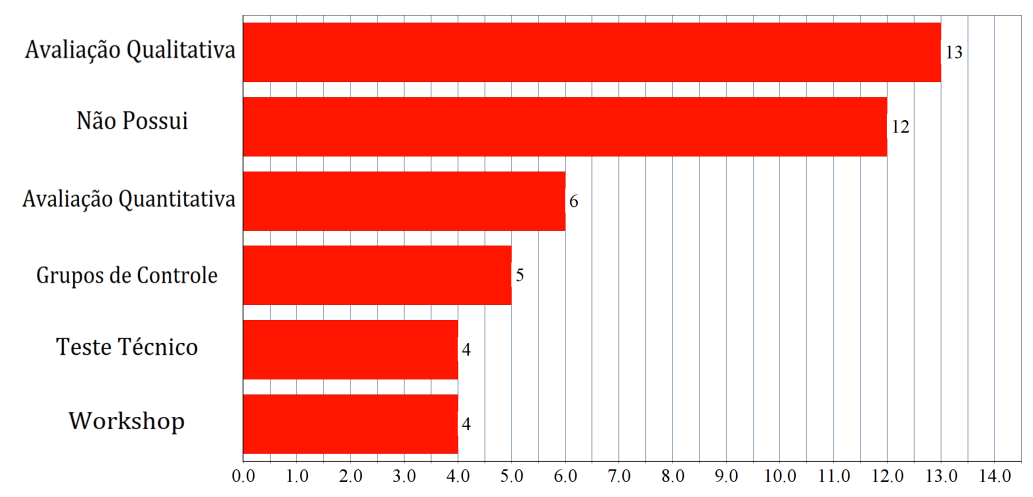

Figura 4. Métodos de Avaliação

No trabalho realizado por Gomez et al. [Gómez et al. 2013] foi possível perceber o uso de grupos de controle no experimento conduzido para avaliar a aplicação IoT desenvolvida. Os autores propuseram um ambiente de aprendizagem no qual os alunos podiam interagir com objetos físicos ao seu redor, os quais estavam virtualmente associados a um conteúdo de aprendizagem. Para avaliar esse sistema, os autores dividiram uma turma de 
VIII Congresso Brasileiro de Informática na Educação (CBIE 2019)

Anais do XXX Simpósio Brasileiro de Informática na Educação (SBIE 2019)

50 alunos em dois grupos. O grupo A participou de uma aula experimental, dentro do ambiente de aprendizagem, enquanto que o grupo B assistiu uma aula tradicional sobre o assunto. Para garantir que ambos os grupos possuíssem o mesmo conhecimento sobre o domínio, um teste prévio foi aplicado e os alunos foram divididos de modo que as médias dos dois grupos fossem iguais.

Após ambos os grupos assistirem suas respectivas aulas, um novo teste foi aplicado. Como resultado, o grupo pertencente à aula experimental conseguiu uma maior média no segundo teste, sugerindo assim uma maior efetividade de aprendizado a partir do uso do sistema desenvolvido pelos autores. Este método se repetiu em outros trabalhos.

Para trabalhos que necessitavam de testes mais técnicos, a pesquisa realizada por Yamada et al. [Yamada et al. 2016] apresenta boas descrições de um Testbed de uma aplicação IoT. Testbed é um ambiente de execução configurado para testes; pode consistir de hardwares específicos, redes, sistemas operacionais, configurações do item a ser testado, entre outros. Um plano de teste de um projeto deve enumerar quais Testbeds serão utilizados [FYICenter ]. Nele, os autores descreveram todas as configurações necessárias para executar a rotina de testes técnicos em uma aplicação e-learning que gerenciava e monitorava o progresso de todos os alunos em um laboratório de informática.

Os autores também testaram o desempenho do protocolo OLSR (Optimized Link State Routing) [Jacquet et al. 2001], um protocolo do tipo ad-hoc para roteamento de redes sem fio. Nessa avaliação, foram analisados: a quantidade máxima de tráfego de dados permitida sem perda de qualidade, o alcance do sinal, a taxa de perda de pacotes para cada um dos alcances possíveis, a capacidade de gerenciar uma rede com diversos dispositivos e a velocidade de transmissão dessas informações. Ao final dos estudos, os autores quantificam e expõe todos estas informações para discussão em futuros trabalhos.

A Figura 5 apresenta a ocorrência das principais tecnologias utilizadas no desenvolvimento de aplicações IoT para educação (QP2) com respeito às áreas de conhecimento (QP1). Dessa maneira, é possível relacionar quais tecnologias apresentam maior utilização em cada área de conhecimento identificada. Em especial, destaca-se a versatilidade das tecnologias RFID tags, NFC e dispositivos móveis, presentes na maioria dos domínios encontrados. Também é possível observar a flexibilidade das disciplinas STEM, cujas aplicações fazem uso de quase todos as tecnologias apresentadas. Por fim, nota-se a ausência das tecnologias de maior ocorrência deste estudo em disciplinas de robótica, o que pode indicar a necessidade de tecnologias mais específicas para a produção de aplicações neste domínio.

\section{Conclusão}

Este trabalho apresentou um mapeamento sistemático conduzido com o intuito de avaliar o estado da arte na área de IoT aplicada à educação. A partir dos resultados obtidos foi possível concluir que: (i) a maioria das aplicações desenvolvidas possuem um foco interdisciplinar e, entre as aplicações que possuem alguma área de conhecimento específica, as disciplinas STEM e de ensino de línguas são as mais abordadas; (ii) dispositivos móveis e RFID tags têm sido as principais tecnologias utilizadas no desenvolvimento de aplicações IoT para educação; e (iii) ainda são poucos os trabalhos que conduzem algum tipo de avaliação das aplicações de IoT para educação; destes, o principal método utilizado é a avaliação qualitativa.

A principal contribuição deste mapeamento sistemático está no conhecimento proporcionado com respeito aos domínios, tecnologias e métodos de avaliação sendo adota- 


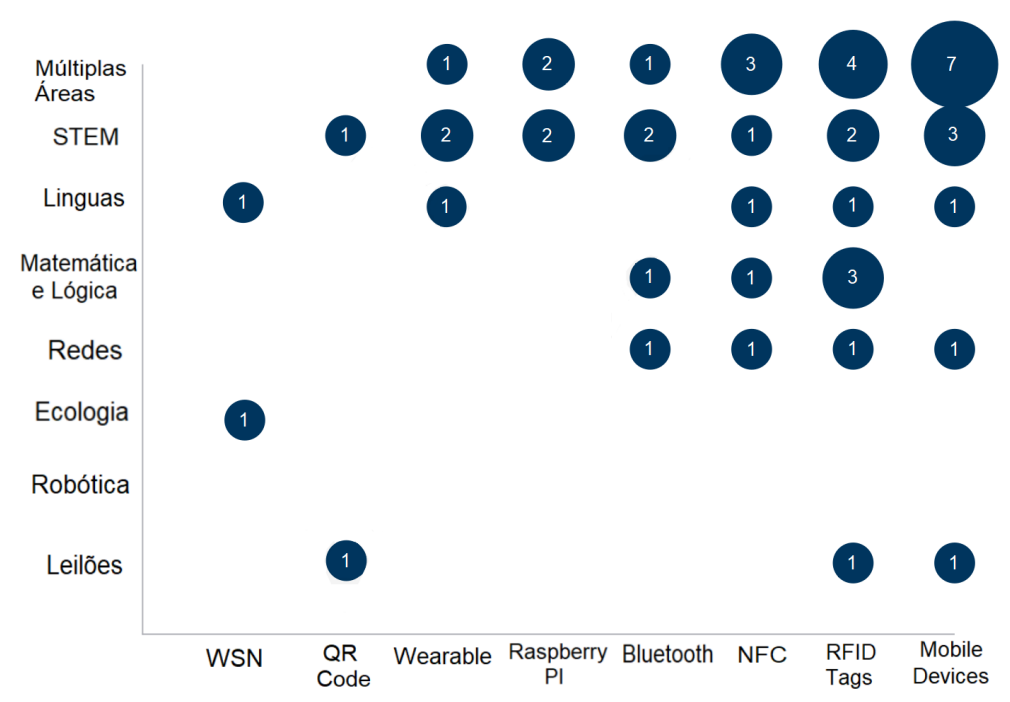

Figura 5. Distribuição das Tecnologias loT em cada Área de Conhecimento

dos no desenvolvimento de aplicações IoT para educação. Ainda, a partir da identificação das atuais tendências e limitações observadas na área, espera-se contribuir para um direcionamento adequado em relação às principais lacunas de pesquisa que requerem maior atenção por parte dos pesquisadores, em curto e médio prazo.

Por fim, como trabalhos futuros, pretende-se investigar aspectos associados ao estabelecimento de processos, métodos e ferramentas que apoiem o projeto, desenvolvimento e avaliação de aplicações IoT para educação, em particular voltadas ao ensino e aprendizagem de fundamentos de programação.

\section{Agradecimentos}

Este estudo foi financiado pela Universidade de São Paulo (USP) e pelas agências Brasileiras de fomento: Coordenação de Aperfeiçoamento de Pessoal de Nível Superior Brasil (CAPES) - Código Financeiro 001/Procad 071/2013, CNPq e FAPESP.

\section{Referências}

Atzori, L., Iera, A., and Morabito, G. (2010). The internet of things: A survey. Computer Networks, 54(15):2787 - 2805 .

Aydin, I. and Othman, N. A. (2017). A new iot combined face detection of people by using computer vision for security application. In 2017 International Artificial Intelligence and Data Processing Symposium (IDAP), pages 1-6.

Chandel, V., Sinharay, A., Ahmed, N., and Ghose, A. (2016). Exploiting imu sensors for iot enabled health monitoring. In Proceedings of the First Workshop on IoT-enabled Healthcare and Wellness Technologies and Systems, IoT of Health '16, pages 21-22, New York, NY, USA. ACM.

Chang, F.-C., Chen, D.-K., and Huang, H.-C. (2015). Future classroom with the internet of things a serviceoriented framework. Journal of Information Hiding and Multimedia Signal Processing, 6(5):869-881. cited By 3.

Charlton, P. and Avramides, K. (2016). Knowledge construction in computer science and engineering when learning through making. IEEE Transactions on Learning Technologies, 9(4):379-390. 
VIII Congresso Brasileiro de Informática na Educação (CBIE 2019)

Anais do XXX Simpósio Brasileiro de Informática na Educação (SBIE 2019)

Correndo, G., Crowle, S., Papay, J., and Boniface, M. (2016). Enhancing marine industry risk management through semantic reconciliation of underwater iot data streams. In Proceedings of the 12th International Conference on Semantic Systems, SEMANTiCS 2016, pages 161-168, New York, NY, USA. ACM.

de la Guía, E., Camacho, V. L., Orozco-Barbosa, L., Luján, V. M. B., Penichet, V. M. R., and Pérez, M. L. (2016). Introducing iot and wearable technologies into task-based language learning for young children. IEEE Transactions on Learning Technologies, 9(4):366-378.

dos Santos, O. L., Cury, D., Rafalski, J., and Silveira, P. D. N. (2016). An iot computational robotics learning laboratory in vila velha, espirito santo. In 2016 XI Latin American Conference on Learning Objects and Technology (LACLO), pages 1-6.

FYICenter. What is test bed? https://goo.gl/ng3Vu7. Acessed: 2017:10:31.

Gómez, J., Huete, J. F., Hoyos, O., Perez, L., and Grigori, D. (2013). Interaction system based on internet of things as support for education. Procedia Computer Science, 21:132 - 139.

Jacquet, P., Muhlethaler, P., Clausen, T., Laouiti, A., Qayyum, A., and Viennot, L. (2001). Optimized link state routing protocol for ad hoc networks. In Proceedings. IEEE International Multi Topic Conference, 2001. IEEE INMIC 2001. Technology for the 21st Century., pages 62-68.

Kitchenham, B. and Charters, S. (2007). Guidelines for performing systematic literature reviews in software engineering. Technical report, Keele University and Durham University Joint Report.

Meda, P., Kumar, M., and Parupalli, R. (2014). Mobile augmented reality application for telugu language learning. In 2014 IEEE International Conference on MOOC, Innovation and Technology in Education (MITE), pages 183-186.

Miglino, O., Di Fuccio, R., Di Ferdinando, A., and Ricci, C. (2015). BlockMagic, A Hybrid Educational Environment Based on RFID Technology and Internet of Things Concepts, pages 64-69. Springer International Publishing, Cham.

Muñoz-Organero, M., Ramírez, G. A., Muñoz-Merino, P. J., and Kloos, C. D. (2011). Framework for Contextualized Learning Ecosystems, pages 260-270. Springer Berlin Heidelberg, Berlin, Heidelberg.

Petersen, K., Vakkalanka, S., and Kuzniarz, L. (2015). Guidelines for conducting systematic mapping studies in software engineering: An update. Information \& Software Technology, 64(C):1-18.

Sula, A., Spaho, E., Matsuo, K., Barolli, L., Miho, R., and Xhafa, F. (2013). An iot-based system for supporting children with autism spectrum disorder. In 2013 Eighth International Conference on Broadband and Wireless Computing, Communication and Applications, pages 282-289.

Tripathi, G. (2016). 5 ways the internet of things is changing education and learning. https://goo.gl/zqrvVN. Acessed: 2017:07:10.

Uzelac, A., Gligoric, N., and Krco, S. (2015). A comprehensive study of parameters in physical environment that impact students' focus during lecture using internet of things. Computers in Human Behavior, $53: 427-434$.

Wang, W., Liu, Y., Guo, Y., and Jian, P. (2016). An educational platform for promoting awareness of lake environmental protection with live monitoring technology. In 2016 International Conference on Educational Innovation through Technology (EITT), pages 238-241.

Wang, Y. (2010). English interactive teaching model which based upon internet of things. In 2010 International Conference on Computer Application and System Modeling (ICCASM 2010), volume 13, pages V13-587-V13-590.

Yamada, M., Oda, T., Liu, Y., Matsuo, K., Ikeda, M., and Barolli, L. (2016). Performance evaluation of an iot-based e-learning testbed considering olsr protocol in a nlos environment. In 2016 19th International Conference on Network-Based Information Systems (NBiS), pages 451-457. 